\title{
Pazopanib is an active treatment in desmoid tumour/aggressive fibromatosis
}

\author{
Juan Martin-Liberal ${ }^{1 *}$, Charlotte Benson ${ }^{1}$, Heather McCarty ${ }^{2}$, Khin Thway $^{1}$, Christina Messiou ${ }^{1}$ and lan Judson ${ }^{1}$
}

\begin{abstract}
Background: Desmoid tumours/aggressive fibromatosis (DT/AF) are infrequent soft-tissue neoplasms. They usually behave as indolent diseases. However, they may grow locally infiltrating or compressing adjacent structures. The role of local treatment is limited and only a few drugs have shown activity.

Cases presentation: We report the outcome of two patients affected by progressive DT/AF treated with the angiogenesis inhibitor pazopanib in two different institutions. Both patients achieved dramatic improvement in their symptoms and radiological signs of response. The clinical benefit lasted for more than 1 year and it is still ongoing.
\end{abstract}

Conclusions: Pazopanib is an active treatment in DT/AF. It is the first time this has been reported.

Keywords: Aggressive fibromatosis, Desmoid tumour, Imatinib, Pazopanib, PDGFR, Pegylated doxorubicin, Sorafenib, VEGFR

\section{Background}

Desmoid tumours/aggressive fibromatosis (DT/AF) are rare soft-tissue neoplasms that usually arise from the abdomen although extremities are also a common site of presentation $[1,2]$. They may be associated with genetic conditions such as Gardner's syndrome [3]. Patients' survival is usually good as DT/AF lack the capacity to metastasize although anatomical disease site is important. Interestingly, the treatment paradigm has changed in the last decade. Aggressive upfront approaches are now under debate since nearly $50 \%$ of patients have relatively indolent disease [4]. Thus, surveillance at initial presentation is the current standard of care in most centres. However, it may become a symptomatic disease as it can grow locally infiltrating or compressing adjacent structures [5].

Surgery is one treatment choice when technically feasible however rates of recurrence and post-treatment morbidity are high [6]. Radiotherapy is also an option but there are real concerns surrounding late effects including development of second malignancies which is important to consider given the young age of most patients. When local treatment with curative intent is not achievable,

\footnotetext{
* Correspondence: juan.martin@rmh.nhs.uk

'The Royal Marsden Hospital, Sarcoma Unit, Fulham Road, SW3 6JJ London, UK

Full list of author information is available at the end of the article
}

systemic treatment should be considered. A widely accepted strategy in first-line setting is hormone treatment, based on the relatively frequent overexpression of oestrogen receptors, used in combination with nonsteroidal anti-inflammatory drugs (NSAIDs) $[7,8]$. If this treatment proves ineffective, cytotoxic chemotherapy is a valid alternative. Pegylated liposomal doxorubicin has shown signs of activity although it is not without toxicity [9]. Several targeted agents have been recently assessed with promising results. The, tyrosine-kinase inhibitor imatinib has been proven to have activity in phase II studies $[10,11]$. Also, recent encouraging data with the antiangiogenic drug sorafenib have been published where the rate of clinical benefit was very high (70\%). In addition, partial response (PR) and stable disease (SD) rates were $25 \%$ and $70 \%$ respectively. Furthermore, $92 \%$ of patients showed features of increased tumour fibrosis and loss of cellularity as demonstrated by an early change in MRI T2 signal [12]. These data support further investigation into the role of antiangiogenic agents in DT/AF.

Pazopanib is one of the latest antiangiogenic drugs developed. It has recently been approved by the U.S. Food and Drug Administration (FDA) and by the European Medicines Agency (EMA) for the treatment of advanced renal cancer and soft-tissue sarcomas (STS). We present 
here the first report of clinical activity of pazopanib in DT/AF. These encouraging results might be the initial step in the development of a new effective therapy this challenging disease.

\section{Cases presentation}

This is the report of the clinical outcome of two patients affected by unresectable DT/AF treated with pazopanib in two different UK institutions: The Royal Marsden Hospital, London and Belfast City Hospital, Belfast. Data were retrospectively collected and imaging was reviewed by a Sarcoma Unit radiologist from The Royal Marsden Hospital.

\section{Patient 1}

Patient 1 is a female who presented at the age of 42 years with a painful mass in the right axilla. A biopsy showed features consistent with DT/AF. The patient did not have personal or family history of Gardner's syndrome or colonic polyps. Surgical resection was performed in two occasions but residual disease remained. As the pain in the axilla increased, treatment with tamoxifen $40 \mathrm{mg}$ once daily (od) was initiated one year after the second surgery. However, there was an increase in the size of the mass and worsening of symptoms in the form of restricted movement and neuropathic pain, so pegylated liposomal doxorubicin was advised. Unfortunately, chemotherapy had to be stopped after one cycle due to an allergic reaction. Subsequent treatment with methotrexate and vinblastine was given. Symptoms were stabilized although the patient experienced disabling toxicities such as severe constipation and neuropathy. After five months, an MRI showed progressive disease (PD), the tumour measuring $15 \times 5.9 \mathrm{~cm}$.

At this point, treatment with pazopanib $800 \mathrm{mg}$ od was started. Shortly afterwards, the patient developed hypertension, dysgeusia and mild palmar erythema so the dose was reduced to $600 \mathrm{mg}$ od. After two months, the patient reported a significant improvement in her pain. Analgesic requirements were dramatically reduced and an MRI showed no changes in size but a significant reduction in tumour T2 signal intensity, suggesting reduction in cellularity. Six months after starting pazopanib, a further dose reduction due to diarrhoea was needed (400 mg od). At that time, a second assessment MRI showed maintained SD and the drop in T2 signal intensity compared to baseline remained suggesting continued response (Figure 1). In the following months, further toxicities appeared: skin rash, peripheral neuropathy and worsening hypertension, dysgeusia and diarrhoea. These side effects led to a further dose reduction to $200 \mathrm{mg}$ od and optimization of supportive treatment.

The patient is currently tolerating pazopanib without significant side effects. Furthermore, her tumour has

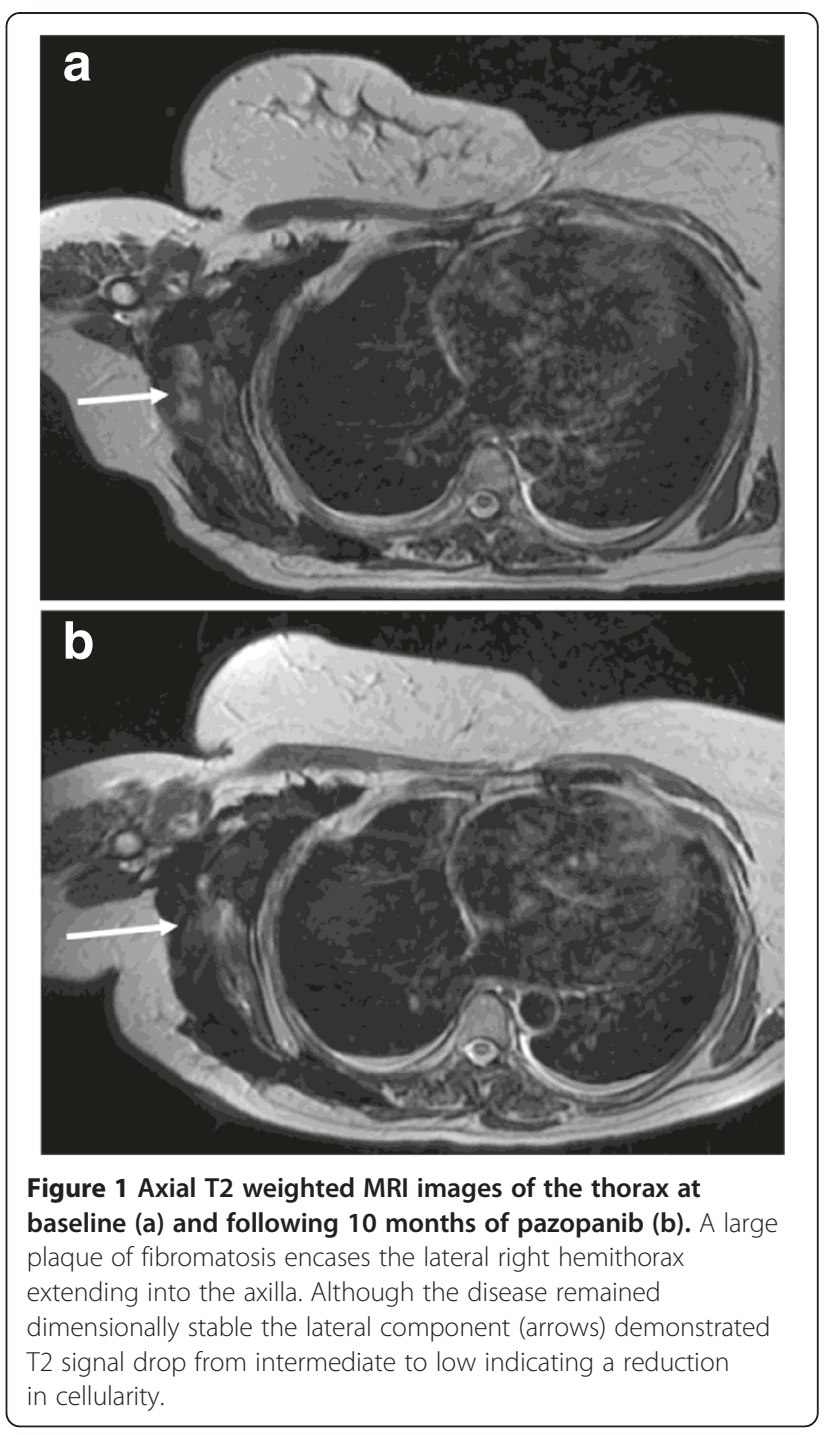

remained stable in size but with T2 changes in MRI suggestive of response for nearly eighteen months. The clinical benefit has been very clear and in particular pain is well controlled, which was the main factor impairing the patient's quality of life.

\section{Patient 2}

Patient 2 is a female who presented at the age of 16 years with a painful left axillary mass. The histological analysis was consistent with DT/AF. The patient did not have a history of Gardner's syndrome or colonic polyps. Incomplete resection was performed and for the following ten years the patient remained asymptomatic. After that time, she developed pain and impairment in the abduction of her left arm. An MRI confirmed tumour recurrence, with involvement of the triceps and inferior deltoid muscles. Surgery was not advised and the patient was started on systemic treatment with high dose NSAIDs; three months on MRI scan showed no significant changes and limitation 
in the abduction of the arm persisted. After one year of treatment, the patient became pregnant and treatment was discontinued. Unfortunately, shortly after the symptoms dramatically worsened. Pegylated liposomal doxorubicin was initiated when the patient was in the third trimester of her pregnancy but with no radiological nor symptomatic benefit. As there were continued significant limitations in arm movement, the patient was very keen to pursue further treatment. Therefore, tumour resection followed by radiotherapy was performed. After these procedures, the patient regained an almost complete range of movement in her arm.

One year after her last operation, an MRI showed two new sites of disease. These findings were associated with worsening of the pain in spite of NSAIDs. Tamoxifen, together with continuation of high dose NSAIDs, was then prescribed. Six months on, further tumour growth and worsening symptoms suggested that the treatment was ineffective.

A new line of treatment with pazopanib $800 \mathrm{mg}$ od was started. After two weeks of treatment, significant
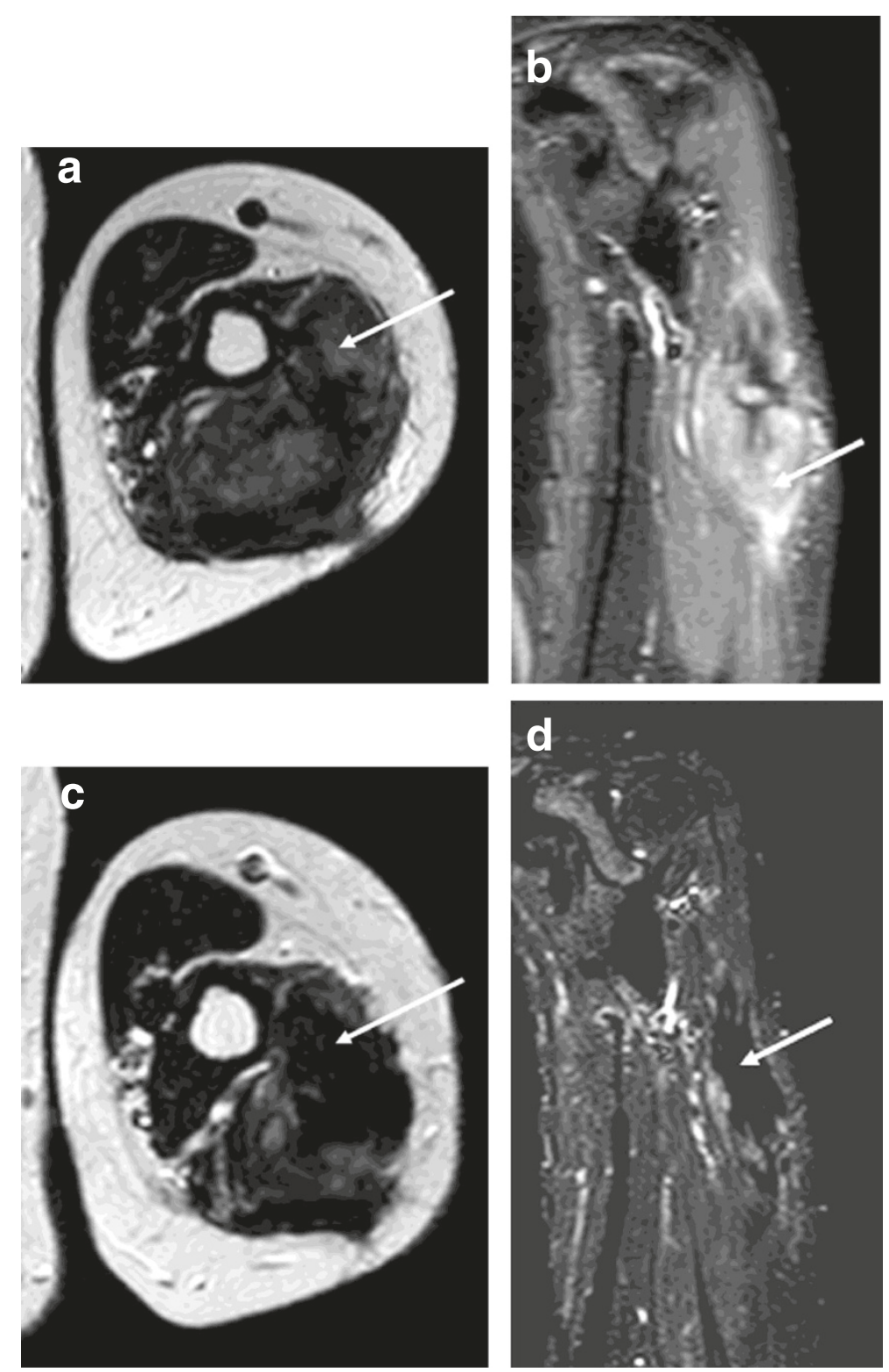

Figure 2 Axial T2 weighted and coronal STIR MRI images of the proximal left arm at baseline ( $a$ and $b$ ) and following 11 months of pazopanib (c and d). A large focus of fibromatosis expands the triceps muscle and following 11 months of treatment reduced in size from $10.2 \mathrm{~cm}$ in maximum craniocaudal dimension to $8.0 \mathrm{~cm}$. Predominantly intermediate/high signal tissue (a and $\mathbf{b}$, arrows) showed a marked reduction in signal (c and $\mathbf{d}$, arrows) indicating diminished cellularity. 
improvement of the pain was noticed. Moreover, the first assessment MRI after three months demonstrated reduction in tumour size (from $10.2 \times 4.2 \mathrm{~cm}$ at baseline to $9 \times 3.1 \mathrm{~cm}$ ). Mild diarrhoea and fatigue were the only side effects. Subsequent MRIs showed further tumour shrinkage and reduction in T2 signal (Figure 2). In addition, mobility of patient's left arm progressively improved. The patient remains on full dose of pazopanib without significant toxicities and with ongoing clinical and radiological benefit more than one year since starting pazopanib.

\section{Conclusions}

This report demonstrates for the first time that pazopanib is an active treatment in DT/AF.

The lack of effective therapeutic options and its high morbidity make DT/AF a challenging disease. The promising results observed with sorafenib suggested that angiogenesis might play an important role in this condition [12]. Although DT/AF is not strictly considered a malignancy, the mechanisms that lead to uncontrolled monoclonal cellular proliferation and survival are similar to those in cancer [13]. Angiogenesis is one of the most important processes in carcinogenesis and its relevance in soft-tissue tumours has also been demonstrated [14]. Several studies confirm the key role of some of its effectors such as the vascular endothelial growth factor (VEGF) and the different isoforms of its receptor (VEGFR) [15-19]. Another crucial protein in the formation of new tumour vessels is the plateletderived growth factor PDGF [20]. Interestingly, both sorafenib and pazopanib inhibit both VEGFR and the PDGF receptor (PDGFR) [21]. Conversely imatinib -a tyrosine-kinase inhibitor which also demonstrates promising activity in DT/AF $[10,11]$ - has effects on PDGFR but not on VEGFR [22]. These data suggest that the efficacy of these drugs in DT/AF might be due to their antiangiogenic activity mediated by the inhibition of different key effectors.

Pazopanib is the first antiangiogenic drug that has shown successful results in a phase III trial in STS [23]. We demonstrate in this report is that it is also active in DT/AF. Furthermore although it has side effects, as shown in Patient 1 , it is generally better tolerated than conventional chemotherapy. This is crucial in a disease such as DT/AF, where curative options are scarce and the principal aim of the treatment is to improve quality of life. In addition, clinical benefit lasted for over one year in both cases and it is still ongoing. Fortunately, the relative lack of cumulative toxicity of pazopanib compared to standard chemotherapy allows an indeterminate duration of treatment.

In conclusion, pazopanib is a promising therapeutic option in DT/AF. However, owing to the retrospective nature of this report and the small number of patients this observation clearly needs to be confirmed in prospective studies. Thus, the French Sarcoma Group is currently conducting a phase II trial that assesses the efficacy and tolerance of pazopanib in DT/AF (ClinicalTrials.gov identifier NCT01876082). This encouraging novel strategy deserves further investigation.

\section{Consent}

Written informed consent was obtained from the patients for publication of this Case Report and any accompanying images. Copies of the written consents are available for review by the Editor-in-Chief of this journal.

\section{Competing interests}

The authors declare that they have no competing interests.

\section{Authors' contributions}

JML, CB, HM and IJ contributed to the conception, design and drafting of the manuscript. CM carried out the radiological evaluation. KT performed the histopathological analyses of the tumours. CB and IJ coordinated the manuscript drafting. All authors read and approved the final manuscript.

\section{Author details}

${ }^{1}$ The Royal Marsden Hospital, Sarcoma Unit, Fulham Road, SW3 6JJ London, UK. ${ }^{2}$ Belfast City Hospital, Lisburn Road, BT9 7AB Belfast, UK.

Received: 12 November 2013 Accepted: 21 November 2013 Published: 26 November 2013

\section{References}

1. Alman BA, Pajerski ME, Diaz-Cano S, Corboy K, Wolfe HJ: Aggressive fibromatosis (desmoid tumor) is a monoclonal disorder. Diagn Mol Pathol 1997, 6(2):98-101.

2. Biermann JS: Desmoid tumors. Curr Treat Options Oncol 2000, 1(3):262-266. 3. Bertagnolli MM, Morgan JA, Fletcher CD, Raut CP, Dileo P, Gill RR, Demetri $\mathrm{GD}$, George S: Multimodality treatment of mesenteric desmoid tumours. Eur J Cancer 2008, 44(16):2404-2410.

4. Bonvalot S, Desai A, Coppola S, Le Péchoux C, Terrier P, Dômont J, Le Cesne A: The treatment of desmoid tumors: a stepwise clinical approach. Ann Oncol 2012, 23(Suppl 10):158-166.

5. Smith AJ, Lewis JJ, Merchant NB, Leung DH, Woodruff JM, Brennan MF: Surgical management of intra-abdominal desmoid tumours. $\mathrm{Br} J$ Surg 2000, 87(5):608-613.

6. Ballo MT, Zagars GK, Pollack A, Pisters PW, Pollack RA: Desmoid tumor: prognostic factors and outcome after surgery, radiation therapy, or combined surgery and radiation therapy. J Clin Oncol 1999, 17(1):158-167.

7. Hansmann A, Adolph C, Vogel T, Unger A, Moeslein G: High-dose tamoxifen and sulindac as first-line treatment for desmoid tumors. Cancer 2004, 100(3):612-620.

8. Deyrup AT, Tretiakova M, Montag AG: Estrogen receptor-beta expression in extraabdominal fibromatoses: an analysis of 40 cases. Cancer 2006, 106(1):208-213.

9. Constantinidou A, Jones RL, Scurr M, Al-Muderis O, Judson I: Pegylated liposomal doxorubicin, an effective, well-tolerated treatment for refractory aggressive fibromatosis. Eur J Cancer 2009, 45(17):2930-2934.

10. Heinrich MC, MCArthur GA, Demetri GD, Joensuu H, Bono P, Herrmann R,

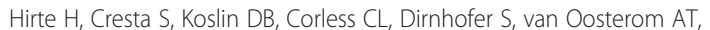
Nikolova Z, Dimitrijevic S, Fletcher JA: Clinical and molecular studies of the effect of imatinib on advanced aggressive fibromatosis (desmoid tumor). J Clin Oncol 2006, 24(7):1195-1203.

11. Penel N, Le Cesne A, Bui BN, Perol D, Brain EG, Ray-Coquard I, Guillemet C, Chevreau C, Cupissol D, Chabaud S, Jimenez M, Duffaud F, PipernoNeumann S, Mignot L, Blay JY: Imatinib for progressive and recurrent aggressive fibromatosis (desmoid tumors): an FNCLCC/French Sarcoma Group phase II trial with a long-term follow-up. Ann Oncol 2011, 22(2):452-457 
12. Gounder MM, Lefkowitz RA, Keohan ML, D'Adamo DR, Hameed M, Antonescu CR, Singer S, Stout K, Ahn L, Maki RG: Activity of Sorafenib against desmoid tumor/deep fibromatosis. Clin Cancer Res 2011, 17(12):4082-4090

13. Li M, Cordon-Cardo C, Gerald WL, Rosai J: Desmoid fibromatosis is a clonal process. Hum Pathol 1996, 27(9):939-943.

14. Martin-Liberal J, Judson I, Benson C: Antiangiogenic approach in soft-tissue sarcomas. Expert Rev Anticancer Ther 2013, 13(8):975-982.

15. Potti A, Ganti AK, Tendulkar K, Sholes K, Chitajallu S, Koch M, Kargas S: Determination of vascular endothelial growth factor (VEGF) overexpression in soft tissue sarcomas and the role of overexpression in leiomyosarcoma. J Cancer Res Clin Oncol 2004, 130(1):52-56.

16. Graeven U, Andre N, Achilles E, Zornig C, Schmiegel W: Serum levels of vascular endothelial growth factor and basic fibroblast growth factor in patients with soft-tissue sarcoma. J Cancer Res Clin Oncol 1999, 125(10):577-581.

17. Hayes AJ, Mostyn-Jones A, Koban MU, A'Hern R, Burton P, Thomas JM: Serum vascular endothelial growth factor as a tumour marker in soft tissue sarcoma. Br J Surg 2004, 91(2):242-247.

18. Chao C, Al-Saleem T, Brooks JJ, Rogatko A, Kraybill WG, Eisenberg B: Vascular endothelial growth factor and soft tissue sarcomas: tumor expression correlates with grade. Ann Surg Oncol 2001, 8(3):260-267.

19. Iyoda A, Hiroshima K, Baba M, Fujisawa T, Yusa T, Ohwada H: Expression of vascular endothelial growth factor in thoracic sarcomas. Ann Thorac Surg 2001, 71(5):1635-1639.

20. George D: Platelet-derived growth factor receptors: a therapeutic target in solid tumors. Semin Oncol 2001, 28(5 Suppl 17):27-33.

21. Négrier S, Raymond E: Antiangiogenic treatments and mechanisms of action in renal cell carcinoma. Invest New Drugs 2012, 30(4):1791-1801.

22. Savage DG, Antman KH: Imatinib mesylate-a new oral targeted therapy. N Engl J Med 2002, 346(9):683-693.

23. van der Graaf WT, Blay JY, Chawla SP, Kim DW, Bui-Nguyen B, Casali PG, Schöffski P, Aglietta M, Staddon AP, Beppu Y, Le Cesne A, Gelderblom H, Judson IR, Araki N, Ouali M, Marreaud S, Hodge R, Dewji MR, Coens C, Demetri GD, Fletcher CD, Dei Tos AP, Hohenberger P, EORTC Soft Tissue and Bone Sarcoma Group, PALETTE study group: Pazopanib for metastatic soft-tissue sarcoma (PALETTE): a randomised, double-blind, placebo-controlled phase 3 trial. Lancet 2012, 379(9829):1879-1886.

doi:10.1186/2045-3329-3-13

Cite this article as: Martin-Liberal et al:: Pazopanib is an active treatment in desmoid tumour/aggressive fibromatosis. Clinical Sarcoma Research 2013 3:13.

\section{Submit your next manuscript to BioMed Central and take full advantage of:}

- Convenient online submission

- Thorough peer review

- No space constraints or color figure charges

- Immediate publication on acceptance

- Inclusion in PubMed, CAS, Scopus and Google Scholar

- Research which is freely available for redistribution 